\title{
Muscle-Specific Receptor Tyrosine Kinase Endocytosis in Acetylcholine Receptor Clustering in Response to Agrin
}

\author{
Dan Zhu, ${ }^{1}$ Zhihua Yang, ${ }^{1}$ Zhenge Luo, ${ }^{2}$ Shiwen Luo, ${ }^{1}$ Wen C. Xiong, ${ }^{1}$ and Lin Mei ${ }^{1}$ \\ ${ }^{1}$ Program of Developmental Neurobiology, Institute of Molecular Medicine and Genetics, Medical College of Georgia, Augusta, Georgia 30912, and \\ ${ }^{2}$ Institute of Neuroscience and Key Laboratory of Neurobiology, Shanghai Institutes for Biological Scineces, Chinese Academy of Sciences, Shanghai 200031, \\ China
}

Agrin, a factor used by motoneurons to direct acetylcholine receptor (AChR) clustering at the neuromuscular junction, initiates signal transduction by activating the muscle-specific receptor tyrosine kinase (MuSK). However, the underlying mechanisms remain poorly defined. Here, we demonstrated that MuSK became rapidly internalized in response to agrin, which appeared to be required for induced AChR clustering. Moreover, we provided evidence for a role of $N$-ethylmaleimide sensitive factor (NSF) in regulating MuSK endocytosis and subsequent signaling in response to agrin stimulation. NSF interacts directly with MuSK with nanomolar affinity, and treatment of muscle cells with the NSF inhibitor $N$-ethylmaleimide, mutation of NSF, or suppression of NSF expression all inhibited agrin-induced AChR clustering. Furthermore, suppression of NSF expression and NSF mutation attenuate MuSK downstream signaling. Our study reveals a potentially novel mechanism that regulates agrin/MuSK signaling cascade.

Key words: neuromuscular junction; nicotinic acetylcholine receptors; agrin; endocytosis; MuSK; NSF

\section{Introduction}

The vertebrate neuromuscular junction (NMJ) is a synapse formed between motoneurons and skeletal muscle fibers. A signature of the NMJ is the accumulation of nicotinic acetylcholine receptors (AChRs) in the postsynaptic membrane. Agrin, a heparan sulfate proteoglycan secreted by the motoneuron (McMahan, 1990), is a factor essential for AChR clustering. Stimulation with agrin causes AChRs to cluster in cultured muscle cells (Nitkin et al., 1987; Campanelli et al., 1991). In agrin mutant mice, motor axons terminate blindly in the muscle with no subjacent AChR clusters at muscle surface in agrin mutant mice (Gautam et al., 1996). Agrin initiates signal transduction events by activating the muscle-specific receptor tyrosine kinase (MuSK) (Jennings et al., 1993; Glass et al., 1996). It rapidly induces MuSK phosphorylation and subsequently activates several intracellular enzymes, including tyrosine kinases such as Abl and Src-family members (Finn et al., 2003; Sadasivam et al., 2005), geranylgeranyltransferase I, a metalloenzyme involved in protein prenylation (Luo et al., 2003), the serine kinase casein kinase 2 (Cheusova et al., 2006), small GTPases of the Rho family (Weston et al., 2000), and p21-activated kinase (PAK) (Luo et al., 2002). In vitro studies indicate a necessary role of these enzymes for agrin-induced AChR clustering. After activation, MuSK binds to adapter proteins such as Dok-7 and ShcD in a tyrosine phosphorylation-

\footnotetext{
Received Sept. 9, 2007; revised Dec. 6, 2007; accepted Dec. 10, 2007.

This work was supported in part by grants from the National Institutes of Health (L.M., W.C.X) and the Muscular Dystrophy Association (L.M.)

Correspondence should be addressed to Lin Mei, Program of Developmental Neurobiology, Institute of Molecular Medicine and Genetics, Medical College of Georgia, CB2803, 1120 15th Street, Augusta, GA 30912. E-mail: Imei@mcg.edu.

D0I:10.1523/JNEUROSCI.4130-07.2008

Copyright $\odot 2008$ Society for Neuroscience $\quad 0270-6474 / 08 / 281688-09 \$ 15.00 / 0$
}

dependent manner (Okada et al., 2006; Jones et al., 2007). Agrin/ MuSK signaling is mediated or regulated by several interacting proteins, including Dishevelled (Dvl) (a protein key in Wnt signaling) and PDZRN3 [a PDZ (postsynaptic density-95/Discs large/zona occludens-1) domain containing RING finger 3 E3 ubiquitin ligase] (Luo et al., 2002; Lu et al., 2007). Dok-7 appears to regulate MuSK activity and propagate signal to downstream effectors; and Dok-7 mutant mice do not form the NMJ (Okada et al., 2006). Dvl is thought to function as an adapter protein to recruit PAK, whereas PDZRN3 was shown to attenuate agrin signaling by degrading surface MuSK (Lu et al., 2007). Although much progress has been made toward identifying the components in the agrin/MuSK signaling cascade, exactly how it leads to AChR clustering remains unclear.

Endocytosis of receptor tyrosine kinases has been shown to be necessary for intracellular signaling in response to various growth factors. Ligand binding promotes the internalization of activated receptors either to attenuate the signaling via degradation of the ligand/receptor complex or to signal from endosomes (Ceresa and Schmid, 2000; Di Fiore and De Camilli, 2001; Wang et al., 2004). Accumulating evidence indicate that ligand-mediated receptor endocytosis is required for signal transduction of receptor tyrosine kinases (Jing et al., 1992; Carpentier, 1994; Vieira et al., 1996; Yang et al., 2005; Liu et al., 2007). However, whether MuSK undergoes ligand-dependent endocytosis has not been investigated. Here, we show that MuSK became rapidly internalized in response to agrin. We provided evidence that agrin-mediated endocytosis of MuSK was required for AChR clustering. Finally, we explored the mechanisms involved in MuSK endocytosis. Our results identified a role of $\mathrm{N}$-ethylmaleimide (NEM) sensitive factor (NSF)-dependent MuSK endocytosis in agrin-induced AChR clustering. 


\section{Materials and Methods}

Materials. $\alpha$-Bungarotoxin ( $\alpha$-BTX), biotin-conjugated $\alpha$-BTX, and Alexa Fluor 594-conjugated $\alpha$-BTX were obtained from Invitrogen (Eugene, OR). Cyanogen bromide-activated Sepharose beads were obtained from GE Healthcare (Piscataway, NJ). $N$-ethylmaleimide was obtained from Sigma (St. Louis, MO). NSF short hairpin RNA (shRNA) constructs were generated by using the BLOCK-iT U6 RNAi Entry Vector kit (Invitrogen, Carlsbad, CA). The sequences of shRNA primers were as follows: shRNA-NSF491, 5' -CAC CGC TTC AAT GAT AAG CTC TTT GCG AAC AAA GAG CTT ATC ATT GAA GC-3' (sense) and 5'-AAA AGC TTC AAT GAT AAG CTC TTT GTT CGC AAA GAG CTT ATC ATT GAA GC-3' (antisense); shRNA-NSF824, 5' -CAC CGC AAA CAT GTT AAA GGC ATC CCG AAG GAT GCC TTT AAC ATG TTT GC-3' (sense) and 5'-AAA AGC AAA CAT GTT AAA GGC ATC CTT CGG GAT GCC TTT AAC ATG TTT GC-3' (antisense); shRNA-NSF1985, 5'-CAC CGC CGC AAG CTT CTT ATC ATC GCG AAC GAT GAT AAG AAG CTT GCG GC-3' (sense) and 5'-AAA AGC CGC AAG CTT CTT ATC ATC GTT CGC GAT GAT AAG AAG CTT GCG GC-3' (antisense). The numbers in shRNA constructs indicate the targeting nucleotide sequences. Primary antibodies were obtained from Upstate Biotechnology (Lake Placid, NY; mouse anti-phosphotyrosine 4G10 and mouse antiRac). Phospho-Abl antibody was obtained from Cell Signaling Technology (Danvers, MA). Anti- $\beta$-AChR antibody (mAb124) were gifts from Dr. J. Lindstrom (University of Pennsylvania Medical School, Philadelphia, PA). Rabbit anti-MuSK antibody was generated using GST-fusion protein containing amino acids 517-569 of mouse MuSK (Luo et al., 2002). Soluble recombinant neural agrin was prepared from COS cells with cDNA encoding the C-terminal half of agrin (Ferns et al., 1993). Anti-NSF antibody was a kind gift from Dr. Richard L. Huganir (Department of Neuroscience and Howard Hughes Medical Institute, Johns Hopkins University School of Medicine, Baltimore, MD).

Cell culture and transfection. Mouse muscle $\mathrm{C} 2 \mathrm{C} 12$ cells were maintained in a nutrient-rich growth medium containing DMEM supplemented with $20 \%$ fetal bovine serum, $0.5 \%$ chicken embryo extract, 2 mM L-glutamine, $100 \mathrm{U} / \mathrm{ml}$ penicillin, and $100 \mathrm{mg} / \mathrm{ml}$ streptomycin at $37^{\circ} \mathrm{C}$ in an atmosphere of $5 \% \mathrm{CO}_{2}$ and $95 \%$ humidity ( $\mathrm{Si}$ et al., 1996; Luo et al., 2002; Zhu et al., 2006). To induce differentiation, myoblasts at $50-70 \%$ confluence were switched to the differentiation medium (DM), DMEM supplemented with $4 \%$ horse serum, and $2 \mathrm{~mm}$ L-glutamine. Transient transfection of $\mathrm{C} 2 \mathrm{C} 12$ cells was performed with lipofectamine 2000 according to the manufacturer instruction (Invitrogen, Carlsbad, CA) with minor modification. Myoblasts were incubated with serumfree DMEM containing plasmids/lipofectamine 2000 regent mixture for at least $16 \mathrm{~h}$ before being switched to DM for 2-3 d. The transfection efficiency could reach $\sim 90 \%$ (Zhu et al., 2006).

Assays of MuSK endocytosis. Endocytosis of MuSK and AChR was assayed using cleavable biotin as described previously (Ehlers, 2000; Yang et al., 2005). Briefly, cells were biotinylated with $1.0 \mathrm{mg} / \mathrm{ml}$ sulfo-NHSSS-biotin (Pierce, Rockford, IL) in DMEM at $4^{\circ} \mathrm{C}$ for $30 \mathrm{~min}$. After washing with cold PBS three times, they were incubated at $37^{\circ} \mathrm{C}$ in DMEM with or without agrin ( $5 \mathrm{nM})$ to allow endocytosis to occur. Cell surface biotin was cleaved by incubation ( $15 \mathrm{~min}$ each, twice) in the glutathione cleavage buffer ( $50 \mathrm{~mm}$ glutathione, $75 \mathrm{~mm} \mathrm{NaCl}, 10 \mathrm{~mm}$ EDTA, $1 \%$ BSA, and $0.075 \mathrm{~N} \mathrm{NaOH}$ ). Cells were washed with PBS three times and scraped into the modified radioimmunoprecipitation assay (RIPA) buffer. Cell lysates were incubated with streptavidin beads on a racking plate overnight at $4^{\circ} \mathrm{C}$. After washing, the beads were mixed with the sample buffer and biotinylated proteins analyzed by immunoblotting. To measure the decrease in MuSK kinases on cell surface, cells were incubated in binding medium (0.01\% BSA in DMEM) with or without agrin $(5 \mathrm{nM})$ at $37^{\circ} \mathrm{C}$ for various times and rinsed with cold DMEM. Surface proteins were biotinylated with $1.0 \mathrm{mg} / \mathrm{ml}$ sulfo-NHS-SS-biotin in DMEM at $4^{\circ} \mathrm{C}$ for $1 \mathrm{~h}$. After washing three times with cold PBS, cells were scraped in the modified RIPA buffer. Biotinylated surface proteins were purified with streptavidin beads and analyzed by Western blot. Amounts of proteins on a blot were quantified by an accompanying standard curve using the NIH Image software.

Yeast two-hybrid screening. A DNA fragment encoding the entire cyto- plasmic domain of mouse MuSK (MuSKic; amino acids 517-871) (Valenzuela et al., 1995) was subcloned in pGBT9 (Clontech, Mountain View, CA) downstream of the Gal4 DNA-binding domain. It was used as bait to screen mouse brain cDNA library in pACT2. Positive clones were selected on plates lacking leucine, tryptophan, and histidine and were further confirmed by a filter assay for $\beta$-galactosidase ( $\beta$-gal) activity as described previously (Huang et al., 2000). To study protein interaction in yeast, ErbB4ic, MuSKic, or MuSK mutants in pGBT9 are cotransformed with NSF, NSF mutants, or the PICK1 (protein kinase C-interacting protein) $\mathrm{c}$ terminus in pGAD424. Transformed yeast cells were seeded in Leu-Trp-His- plates and scored for $\beta$-gal activity: $(-)$, no blue after $8 \mathrm{~h}$; $(+)$, blue after $2 \mathrm{~h} ;(++)$, blue within $1 \mathrm{~h}$.

Immunofluorescence confocal microscopy. Denervated ( $5 \mathrm{~d}$ postdenervation) muscle sections were fixed in $4 \%$ paraformaldehyde and incubated with $2 \%$ normal goat serum (Vector Laboratories, Burlingame, $\mathrm{CA}$ ) in PBS for $1 \mathrm{~h}$ at room temperature to reduce background staining. Sections were then incubated with anti-NSF antibody (1:1000) in $2 \%$ normal goat serum/PBS at $4^{\circ} \mathrm{C}$ overnight. After washing three times, the samples were incubated with Alexa 488-conjugated anti-rabbit antibody and Alexa 594-conjugated $\alpha$-BTX to label AChRs. Images were collected on a Zeiss (Jena, Germany) confocal microscope.

Immunoprecipitation and immunoblotting. $\mathrm{C} 2 \mathrm{C} 12$ myoblasts were transfected with plasmid DNA with lipofectamine 2000. Three days after transfection, myotubes were treated with or without agrin $(5 \mathrm{~nm}, 30$ $\mathrm{min}$ ) and rinsed with ice-cold PBS and solubilized for $15 \mathrm{~min}$ on ice in modified RIPA buffer supplemented with the protease inhibitors. Lysates were then centrifuged at $12,000 \mathrm{rpm}$ for $10 \mathrm{~min}$, and the supernatants were immunoprecipitated at $4^{\circ} \mathrm{C}$ overnight with NSF antibody followed by protein A beads. After centrifugation, beads were washed four to five times with the lysis buffer. Bound proteins were eluted with SDS sample buffer and subjected to SDS-PAGE. Proteins resolved on SDS-PAGE were transferred to nitrocellulose membranes (Schleicher and Schuell, Keene, NH). Nitrocellulose blots were incubated at room temperature for $1 \mathrm{~h}$ in blocking buffer (TBS with $0.1 \%$ Tween 20 and 5\% milk), followed by an incubation with indicated antibodies at $4^{\circ} \mathrm{C}$ overnight. After washing three times for 15 min each with TBS with $0.1 \%$ Tween 20 , the blots were incubated with horseradish peroxidase-conjugated secondary antibody. (GE Healthcare) followed by another wash. Immunoreactive bands were visualized using enhanced chemiluminescence substrate (Pierce, Rockford, IL). All immunoblotting was repeated at least three times.

\section{Results}

\section{Agrin induces rapid internalization of MuSK}

To study agrin/MuSK signaling mechanisms, we determined whether MuSK internalizes in response to agrin stimulation in C2C12 cells that express endogenous MuSK. Cells were incubated with sulfo-NHS-SS-biotin at $4^{\circ} \mathrm{C}$ to label surface proteins. After washing off free biotin, cells were incubated at $37^{\circ} \mathrm{C}$ to allow endocytosis to occur. Cell surface biotin was then cleaved by incubation in the glutathione cleavage buffer. Biotinylated proteins were purified by streptavidin beads and analyzed by Western blot. As shown in Figure $1 A$, MuSK endocytosis was minimal in control cells but increased dramatically in cells that were stimulated with agrin. Quantitative analysis showed that MuSK endocytosis was fast with $36 \pm 5.5 \%$ internalized within a few minutes of stimulation (Figs. $1 B, 7 D$ ). In contrast, the amount of internalized AChRs was barely detectable, in either control or agrin-treated myotubes (Fig. $1 A$ ). To determine whether internalized MuSK comes from cell surface, we monitored surface MuSK after agrin stimulation. In this assay, surface AChR after biotinylation was not cleaved with the glutathione cleavage buffer. In the absence of agrin, surface MuSK levels changed little for $30 \mathrm{~min}$ observed. However, ligand stimulation caused a rapid decrease in surface MuSK (Fig. 1A); the time course of the decrease was apparently reciprocal of the increase in internalized MuSK (Fig. 1A). Surface levels of AChRs remained the same in 


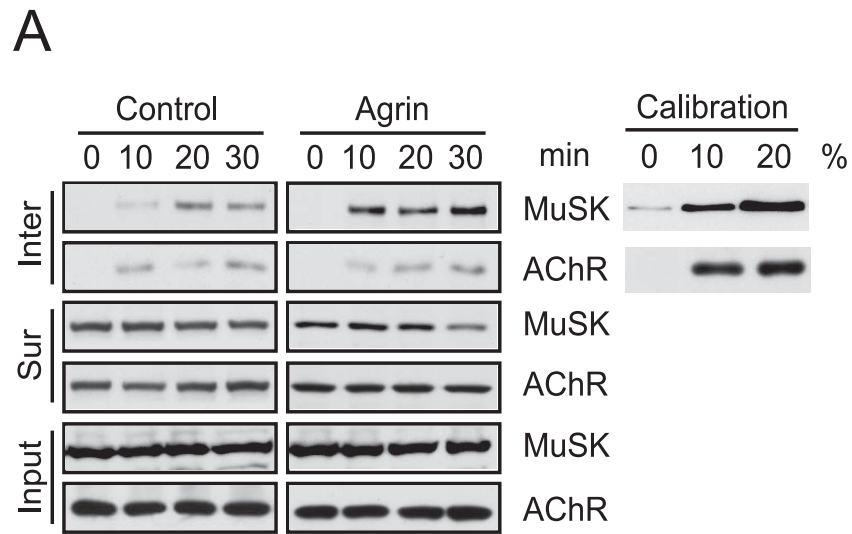

B

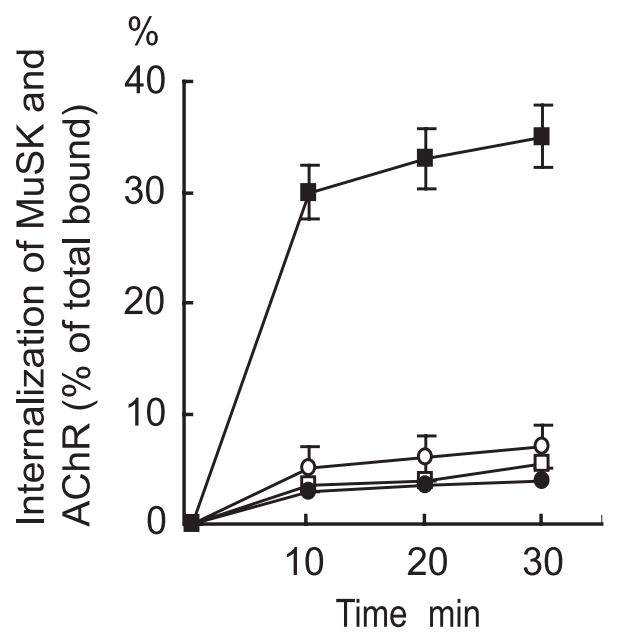

Figure 1. Agrin induces rapid internalization of MuSK. A, MuSK endocytosis in C2C12 cells in response to agrin stimulation. Cells were incubated with sulfo-NHS-SS-biotin to biotinylate surface proteins and subsequently challenged without or with agrin $(5 \mathrm{~nm})$ for indicated times. Cells were then incubated with the glutathione buffer to cleave biotin that remained on the cell surface. Internalized biotinylated proteins (Inter; top panels) were purified with streptavidin beads and analyzed by immunoblotting with anti-MuSK and anti-AChR antibodies. To monitor surface proteins, $\mathrm{C2}$ C12 cells were stimulated without or with agrin and incubated with sulfoNHS-SS-biotin. Biotinylated surface proteins (Sur; middle panels) were purified with streptavidin beads and analyzed by immunoblotting. Bottom panels (Input) show equal amounts of MuSK and AChR in cell lysates as loading control. $\boldsymbol{B}$, Quantitative analysis of data in $\boldsymbol{A}$. Scanned autoradiograms were analyzed by the NIH Image software. The amounts of internalized proteins were calibrated with a standard curve of total biotinylated proteins (inset, right panels in A). Data shown were means \pm SEM of three independent experiments. Squares, MuSK; circles, AChR (open, control; closed, stimulated).

both control and agrin-treated cells (Wallace, 1992; Borges and Ferns, 2001; Zhu et al., 2006), consistent with previous observations that AChR internalization was not detectable in $\mathrm{C} 2 \mathrm{C} 12$ cell culture in vitro (Bruneau et al., 2005). Together, these results indicate that MuSK undergoes endocytosis after agrin stimulation, very much like other receptor tyrosine kinases such as EGFR (epidermal growth factor receptor) and ErbB4, which are known to internalize after ligand stimulation (Vieira et al., 1996; Yang et al., 2005).

Inhibition of MuSK endocytosis attenuated $\mathrm{AChR}$ clustering To study the function of MuSK endocytosis, we explored the consequences of expression of $\mathrm{K} 44 \mathrm{~A}$, a dominant-negative mutant of dynamin. Dynamin is a GTPase that liberates nascent
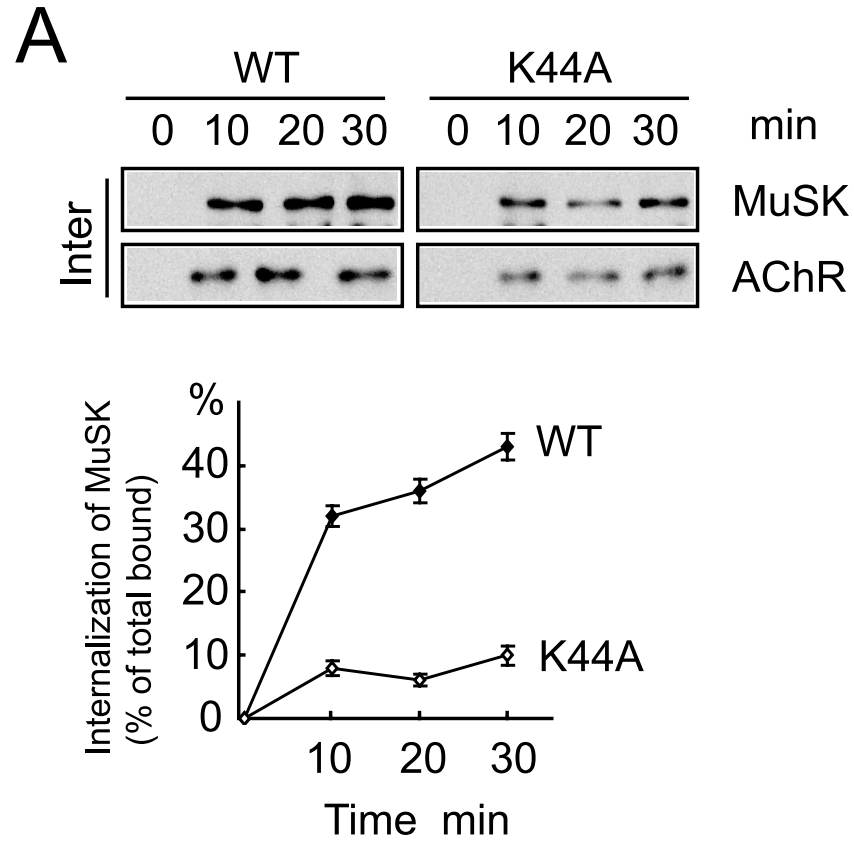

B

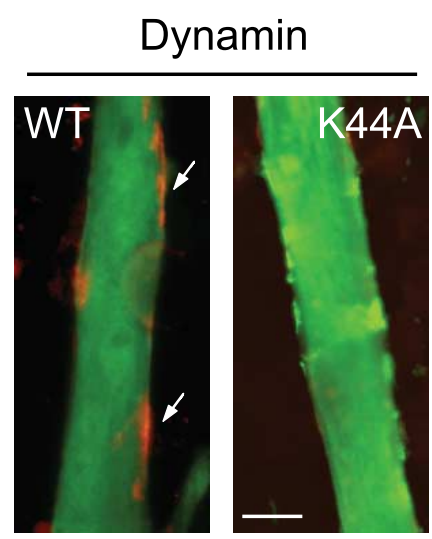

Figure 2. Inhibition of MuSK endocytosis attenuates AChR clustering. $\boldsymbol{A}$, Dynamin K44A attenuated MuSK endocytosis. 22 C12 cells were transfected with HA-tagged wild-type or K44A dynamin. MuSK endocytosis was assayed as in Figure 1. B, Dynamin K44A attenuated AChR clustering. $\mathrm{C}_{2} \mathrm{C} 12$ cells were transfected with HA-tagged wild-type or K44A dynamin along with EGFP expressing construct. Fully differentiated myotubes were treated with agrin (1 nм; $16 \mathrm{~h}$ ) and then were incubated with Alexa 594-BTX to stain AChR clusters (arrows). Myotube segments (200 $\mu \mathrm{m}$ in length) were viewed at $40 \times$ magnification with a Nikon (Tokyo, Japan) Optiphot microscope equipped with phase and epifluorescence optics, and the number of AChR aggregates was counted. AChR clusters with the shortest axis, $>4 \mu \mathrm{m}$, were counted in 10 fields of each experiment. Scale bar, $20 \mu \mathrm{m}$.

clathrin-coated pits from the plasma membrane during endocytosis (Danino and Hinshaw, 2001). Previous studies have shown that K44A inhibits endocytosis of various transmembrane proteins (Vieira et al., 1996; Yang et al., 2005). As shown in Figure 2 A, dynamin K44A, but not wild-type (WT) dynamin, inhibited agrin-induced MuSK endocytosis. We next examined whether MuSK endocytosis is necessary for agrin-induced AChR clustering. $\mathrm{C} 2 \mathrm{C} 12$ myoblasts were cotransfected with enhanced green fluorescent protein (EGFP)-fusion proteins of WT and K44A dynamin. Twenty-four hours after transfection, cells were switched to differentiation medium. Fully differentiated myotubes were stimulated with agrin $(1 \mathrm{~nm}, 16 \mathrm{~h})$ to induce AChR clusters. Our previous study indicated that expression of dy- 
A

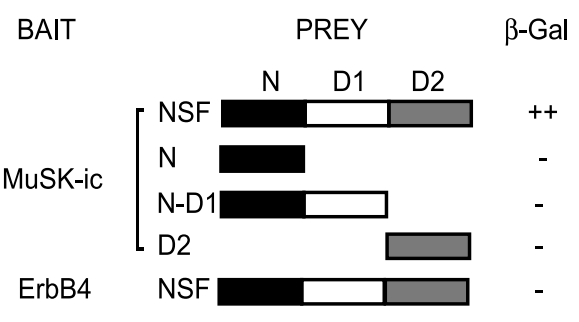

C

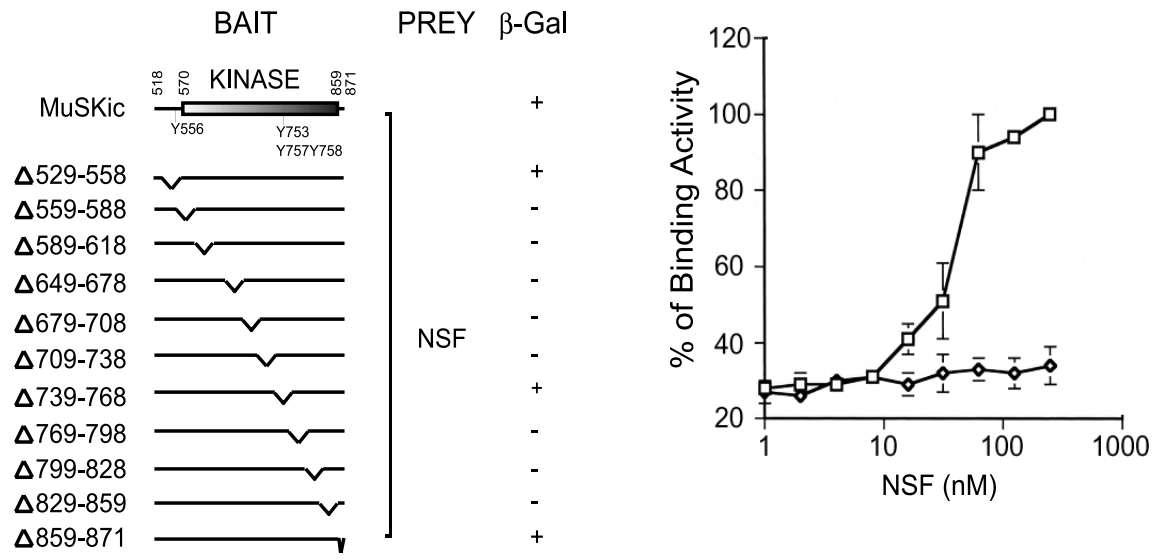

E
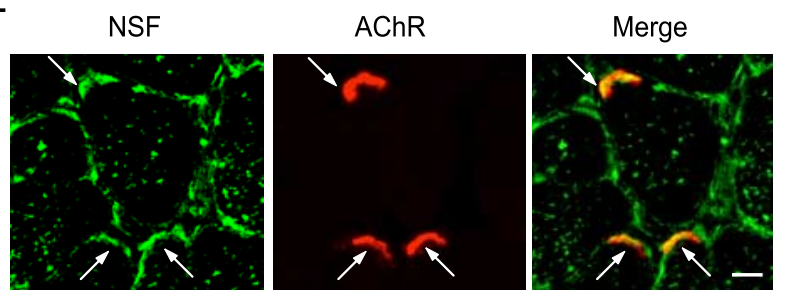

Figure 3. Interaction of MuSK and NSF in yeast. $\boldsymbol{A}$, Interaction of MuSK and NSF requires the full length of NSF. The domain structure of NSF is shown in the schematic diagram. The original clone isolated from a yeast two-hybrid screen encodes the full-length NSF. The N-terminal domain (amino acids 1-211), N-D domain (amino acids $1-495$ ), and $\mathrm{D}_{2}$ domain (amino acids 496 -752) were subcloned in pGAD424, fused to C terminus of Gal4AD. These constructs were cotransformed with Gal4BD/ MuSKic. Transformed yeast cells were seeded in -Leu-Trp-His plates and scored for $\beta$-gal activity. $\boldsymbol{B}$, Dependence on the MuSK kinase domain for the interaction. Various domains of MuSKic were subcloned in pGBT9 and were cotransformed with Gal4AD/ NSF. Interaction was determined as in A. C, NSF interacts with a series of MuSKic mutants with a deletion of 30 aa in the juxtamembrane, kinase, or C-terminal domains. D, Direct interaction between MuSK and NSF. The affinity between MuSK and NSF was determined by ELISA assay. Recombinant MuSK protein was coated in a 96-well plate and incubated with increasing concentrations of GST-NSF fusion proteins or GST alone as negative controls. Bound NSF was detected by monoclonal anti-GST antibody, followed by anti-mouse secondary antibody conjugated to alkaline phosphatase. $\boldsymbol{E}$, NSF immunoreactivity was detected in AChR clusters as well as in sarcolemma. Denervated muscle sections were incubated with the anti-NSF antibody followed by Alexa 488-conjugated secondary antibody and Alexa 594-conjugated $\alpha$-BTX. Scale bar, $20 \mu \mathrm{m}$. Arrows, NMJ.

Identification of NSF as a MuSKinteracting protein by a yeast two-hybrid screen

To explore the mechanisms underlying MuSK endocytosis, we applied yeast twohybrid system to identify proteins that bind to MuSK. A yeast two-hybrid system screen of mouse cDNA libraries $\left(5 \times 10^{6}\right.$ clones $)$ using the MuSK entire cytoplasmic domain as bait led to the finding of 119 positive clones, several of which encoded mouse NSF with a deletion of the $\mathrm{N}$-terminal 7 amino acids. NSF is a protein implicated in membrane fusion and protein trafficking. It contains three distinct domains: $\mathrm{N}$ terminal ( $\mathrm{N}$ domain, residues 1-211), $\mathrm{D}_{1}$ domain (residues 212-495), and $\mathrm{D}_{2}$ domain (residues 496-752) (Tagaya et al., 1993). The NSF-MuSK binding was specific in that NSF did not bind to the intracellular portion of ErbB4, a nonrelated receptor tyrosine kinase (Fig. $3 A$ ). It appeared to require a proper folding or full length of NSF, because deletion of any of the three domains prevented NSF from interacting with MuSK (Fig. 3A). We characterized the interaction between NSF and MuSK deletion mutants. As shown in Figure $3 B$, only the mutants containing the intact kinase domain can bind to NSF. To map which region in MuSK was essential for interaction, we generated a battery of MuSK mutants, each with deletion of 30 amino acids in the intracellular domain. Deletion of the $\mathrm{C}$ terminus (13 amino acids) had no effect on MuSK binding to NSF, suggesting that this region and PDZdependent mechanism were not required for interaction (Fig. 3C). Deletion of the middle 30 amino acids in the juxtamembrane, including Y556, a tyrosine implicated in agrin signaling (Zhou et al., 1999; Herbst and Burden, 2000; Okada et al., 2006), had no apparent effect on binding to NSF. In contrast, deletion of any 30 amino acids in the kinase domain or in the region linking the juxtamembrane and kinase domains abolished the binding activity, with the exception of one deletion mutant, $\Delta 739-768$. To determine whether NSF and MuSK directly interact and not via a third protein, recombinant MuSK was coated in a 96-well plate and incubated with increas-

namin K44A did not inhibit myotube formation (Yang et al., 2005). As shown in Figure $2 B$, expression of dynamin K44A in $\mathrm{C} 2 \mathrm{C} 12$ cells greatly attenuated AChR clustering, and the AChR clusters became smaller and fragmented (in WT-transfected myotubes, $61.07 \pm 6.14$ clusters/field; in K44A-transfected myotubes, $17.63 \pm 3.26$ clusters/field). The number of clusters larger than $4 \mu \mathrm{m}$ was scored, and data were averaged from three different experiments and shown as mean \pm SEM $\left({ }^{*} p<0.01\right)$. These results indicate that MuSK endocytosis may be required for agrin signaling and AChR clustering. ing concentrations of GST-NSF fusion proteins. Bound NSF was detected by monoclonal anti-GST antibody, followed by antimouse secondary antibody conjugated to alkaline phosphatase. As shown in Figure 3D, NSF bound to MuSK in dose-dependent and saturable manners with an $\mathrm{EC}_{50}$ value of $46 \pm 4 \mathrm{~nm}$. In contrast, GST protein alone did not bind to MuSK. These results demonstrate that MuSK interacts with NSF directly and specifically with high affinity. To determine subcellular localization of NSF in skeletal muscle and to eliminate possible contamination from presynaptic terminals where NSF is enriched, we stained 
A

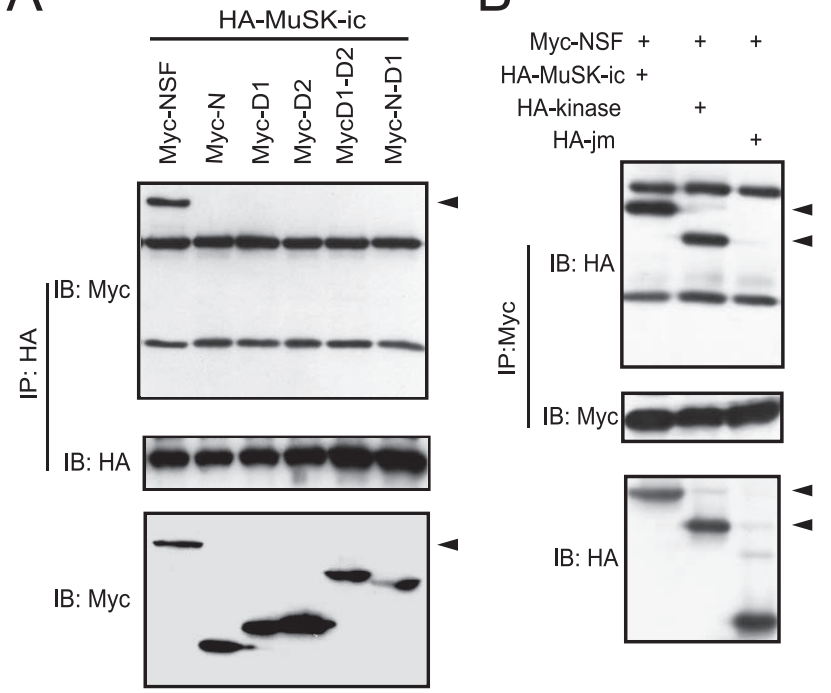

Figure 4. Interaction of MuSK and NSF in mammalian cells. $A$, Identification of the domain in NSF to interact with MuSK. Various NSF constructs were generated by PCR and subcloned in pCMV with a Myc tag in the N terminus. N, Amino acids $1-211 ; D_{1}$, amino acids $211-495 ; D_{2}$, amino acids $496-752 ; \mathrm{D}_{1}-\mathrm{D}_{2}$, amino acids $211-752 ; \mathrm{N}-\mathrm{D}_{1}$, amino acids $1-495$. The intracellular domain of MuSK (amino acids 515-868) was subcloned in pKH3 vector with an HA tag in the N terminus. HEK293 cells were cotransfected with indicated constructs, and $48 \mathrm{~h}$ after transfection, cells were lysed and subjected to immunoprecipitation (IP) with anti-HA antibody. Cell lysates were examined for the expression of NSF constructs (bottom panel). IB, Immunoblot. $\boldsymbol{B}$, The kinase domain is sufficient for the interaction. Various MuSK constructs were generated by PCR and subcloned in pKH3 vector. HEK293 cells were cotransfected with indicated constructs, and cell lysates were subjected to immunoprecipitation with anti-Myc antibody.

sections of denervated leg muscles (postdenervation day 5). Sections were stained with anti-NSF antibody and Alexa 594conjugated $\alpha$-BTX to label AChRs. The immunoreactivity of NSF was visualized with an Alexa 488-conjugated secondary antibody. As shown in Figure 3E, NSF immunoreactivity was present in the sarcolemma of the skeletal muscle but also colocalized with $\alpha$-BTX. This notion was supported by overlapping staining in merged images. These results indicated that NSF is enriched at the NMJ.

To determine whether MuSK interacts with NSF in mammalian cells, we coexpressed HA-tagged MuSK with Myc-tagged NSF wild-type or mutant constructs in human embryonic kidney 293 (HEK293) cells. Forty-eight hours after transfection, cells were harvested, and cell lysates were immunoprecipitated with anti-HA antibodies followed by immunoblotting with anti-Myc antibody. NSF was readily detected in immunoprecipitates in cells coexpressing MuSK (Fig. 4A), suggesting that MuSK associates with NSF in vivo. In agreement with yeast studies, only full length of NSF was coprecipitated with MuSK (Fig. 3A), and NSF was associated with intracellular or kinase domain of MuSK but not the juxtamembrane domain (Fig. $4 B$ ).

\section{Involvement of NSF in AChR clustering}

If the MuSK-NSF interaction is important for agrin signaling, its disruption should inhibit AChR cluster formation. To test this hypothesis, C2C12 myotubes were treated with NEM, a chemical that inactivates NSF (Malhotra et al., 1988). Before investigating the effect of NEM on AChR clustering, we determined its effect on myotube viability. Quantitative analysis using trypan blue exclusion indicated that the percentage of dead myotubes after methyl cyclodextrin (MCD) treatment $(50 \mathrm{nM}, 24 \mathrm{~h})$ was the same as control $(\sim 1 \%)$. Furthermore, there was no difference in morphology between control and NEM-treated myotubes (data not shown). Thus, NEM did not affect the viability of cultured $\mathrm{C} 2 \mathrm{C} 12$ cells under these conditions. $\mathrm{C} 2 \mathrm{C} 12$ myotubes were pretreated with NEM for $6 \mathrm{~h}$ and then incubated with agrin for another $16 \mathrm{~h}$. As shown in Figure 5, agrin-induced AChR aggregates were impaired in $\mathrm{C} 2 \mathrm{C} 12$ myotubes in the presence of NEM. The effect did not appear to be nonspecific, because it is concentration dependent (Fig. 5). These results suggested that the activity of NSF may be required for the agrin/MuSK signaling cascade.

Next, a DNA-based shRNA approach was used to suppress NSF expression in $\mathrm{C} 2 \mathrm{C} 12$ muscle cells to determine whether AChR clustering requires NSF. Three shRNA-NSF constructs were tested for effectiveness to inhibit NSF expression (Fig. 6A). shRNA-NSF491 (shRNA-491) was most effective in inhibiting expression of endogenous NSF in C2C12 cells, followed by shRNA-824. shRNA-1985 had no effect and was used as control in further study. As a negative control, an shRNA construct containing the scrambled sequence had no effect on NSF expression (Fig. 6A). Importantly, the effect of shRNA-491 was specific, because it did not affect the expression of MuSK (Fig. 6A). We next tested whether knockdown of NSF would inhibit myotube formation. Myoblasts were transfected with either control scrambled shRNA or shRNA-491 along with EGFP expressing construct; $24 \mathrm{~h}$ later, cells were switched to DM to induce differentiation. As shown in Figure 6B, no difference was found between control and NSF-shRNA-transfected myotubes. Moreover, shRNA-491 and shRNA-824 had no effect on expression of myogenin (Fig. 6A), a key molecule for muscle differentiation. These results indicated that inhibition of NSF expression did not affect myotube formation. We then characterized the AChR clusters in the presence of shRNA-NSF; shRNA constructs were introduced into $\mathrm{C} 2 \mathrm{C} 12$ myoblasts together with a plasmid encoding EGFP to label transfected cells. Resulting myotubes were stained with Alexa-594 BTX and analyzed for AChR clusters in response to agrin (Fig. 6C). The number of clusters in shRNA-491transfected myotubes $(16.09 \pm 3.12$ clusters/field) was reduced compared with control or shRNA-1985-transfected myotubes $\left(57.11 \pm 6.18\right.$ clusters/field; mean \pm SEM; $\left.n=30 ;{ }^{\star} p<0.01\right)$. These results indicate a necessary role of NSF in this event.

\section{NSF mutation attenuated MuSK endocytosis, AChR clustering, and agrin signaling}

Our finding that NEM inhibited AChR clustering suggests to us that NSF activity may be required for agrin signaling. To inhibit NSF activity, we mutated the glutamic acid residue at 329 in the catalytic site of the $\mathrm{D}_{1}$ domain to glutamine (E329Q) (Coppolino et al., 2001). E329Q-NSF forms hexamers that retain ATP binding activity but is unable to hydrolyze it (Whiteheart et al., 1994; Ward et al., 1997). Wild-type and dominant-negative NSF constructs were transfected in $\mathrm{C} 2 \mathrm{C} 12$ cells, and the interaction of MuSK and NSF was assayed. As shown in Figure 7A, E329Q-NSF was impaired in binding to MuSK, suggesting that the ATPase activity is required for the association. Intriguingly, agrininduced MuSK endocytosis was reduced in C2C12 myotubes expressing E329Q-NSF (Fig. 7B). Note that this effect was specific, because E329Q-NSF had little effect on AChR endocytosis. These results suggest that NSF and its ATPase activity are necessary for induced MuSK endocytosis. In support of this notion was the observation that agrin-induced AChR clustering was attenuated in myotubes expressing E329Q (Fig. 7C). These results and those from NEM studies are in good agreement, suggesting that NSF inhibition disrupts AChR clustering. Next, we determined whether NSF internalized together with MuSK. As shown in Fig- 

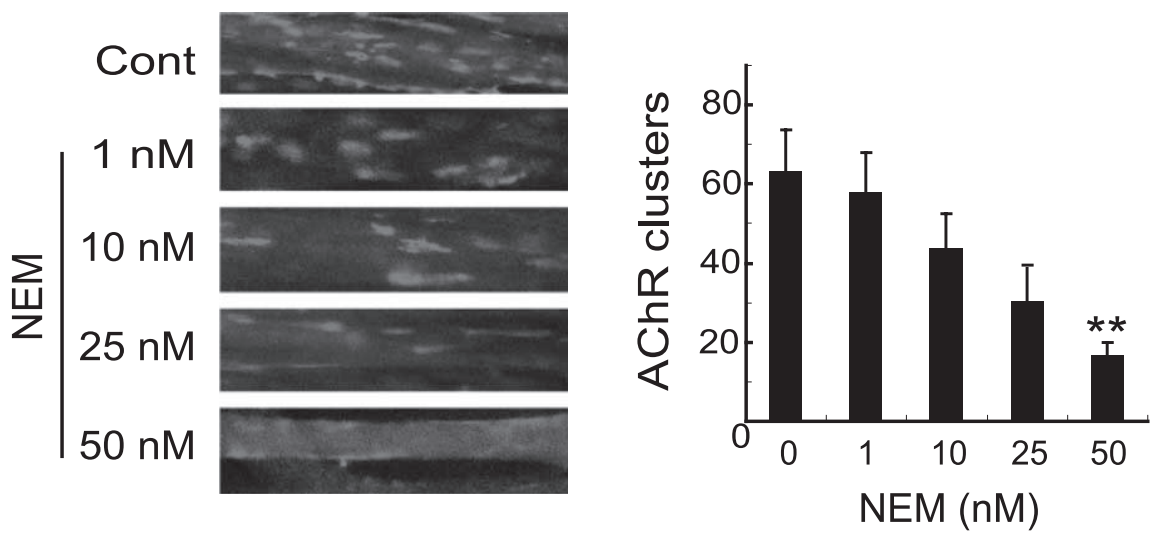

Figure 5. NEM treatment inhibits agrin-induced AChR clusters. C2C12 myotubes were pretreated with or without 1, 10, 25, and $50 \mathrm{~nm}$ NEM for $6 \mathrm{~h}$ before stimulation with agrin ( $1 \mathrm{~nm} ; 16 \mathrm{~h}$ ). NEM remained in the medium during the entire course of agrin incubation. AChR clusters were assayed as in Figure 2. Histograms show quantification of AChR clusters (mean $\pm S E M ; n=30$; $\left.{ }^{*} p<0.01\right)$. Cont, Control.

ure $7 D$, agrin induced rapid internalization of MuSK; however, NSF was not detectable with endocytosed MuSK, suggesting that NSF may dissociate from MuSK after its endocytosis.

To further explore mechanisms of NSF regulation of agrin activity, we characterized effects of suppressing NSF expression and mutation of its ATPase activity on previously identified signaling events. We first examined MuSK tyrosine phosphorylation in the presence of shRNA-NSF or E329Q-NSF. Agrin (5 nM) increased tyrosine phosphorylation of MuSK within $30 \mathrm{~min}$ in control, but not in shRNA-491- or E329Q-NSF-expressing myotubes (Fig. $8 \mathrm{~A}$ ). These results suggested that an appropriate level of NSF and its ATPase activity may be required for MuSK endocytosis and subsequent activation. Activation of MuSK induces rapid phosphorylation of Abl, which is required for AChR clustering (Finn et al., 2003). Abl phosphorylation was determined in myotubes expressing shRNA-491 or E329Q-NSF. As shown in Figure $8 B$, agrin-stimulated Abl phosphorylation was attenuated in myotubes expressing shRNA-491 or E329Q-NSF, indicating that NSF functions above Abl. We also determined effects on the activation of Rho family GTPases, another downstream step. The amount of active Rac1, purified by GST-p21-binding domain (PBD) immobilized on beads, was revealed by Western blotting. In control cells, agrin treatment elicited an approximately fivefold increase in active Rac1 (Fig. 8C); however, agrin-induced Racl activation was inhibited in myotubes expressing shRNA491 or E329Q-NSF. These results suggest again that NSF is necessary for activation of MuSK and subsequent downstream signaling.

\section{Discussion}

In the present study, we demonstrate that agrin stimulates MuSK endocytosis, which appeared to be required for induced AChR clustering. Moreover, we provided evidence for a role of NSF in regulating MuSK endocytosis and subsequent signaling in response to agrin stimulation. First, NSF interacts directly with MuSK with a nanomolar affinity. Second, treatment of muscle cells with the NSF inhibitor NEM, mutation of NSF, and suppression of NSF expression inhibit agrin-induced AChR clustering. Third, suppression of NSF expression and NSF mutation attenuate agrin signaling, including MuSK tyrosine phosphorylation, Abl activation, and activities of Rho GTPases. Our study reveals a potentially novel mechanism that regulates agrin/MuSK signaling cascade.

Endocytosis and trafficking of receptor tyrosine kinases has been shown to be important for initiating and controlling downstream signaling pathways. Endocytosis of activated Trk receptors is necessary for some biological functions of neurotrophins in neurons (Riccio et al., 1997; Zhang et al., 2000). Internalized Trk kinases could remain tyrosine phosphorylated and active, with extracellular domains interacting with cointernalized growth factors inside signaling endosomes. This mechanism is thought to be key to sustained activation of Erk and PI3 kinase (Beattie et al., 2000; Howe et al., 2001). In terms of neuregulin-1 signaling, we have shown that endocytosis of ErbB receptors are required for signal transduction (Yang et al., 2005; Liu et al., 2007). In the current study, we studied MuSK receptor endocytosis by labeling membrane surface protein with biotin. We provide evidence that MuSK proteins underwent rapid endocytosis in response to agrin in muscle cells. Endocytosed MuSK proteins were detectable within 10 min of stimulation. More than $36 \%$ of total surface MuSK became internalized within $30 \mathrm{~min}$ (Fig. 1).

Endocytosis, characterized by internalization of molecules from cell surface into internal membrane compartments, can be divided into two main pathways: the classic, clathrin-mediated endocytic pathway; and the nonclassic, clathrin-independent but lipid-raft-dependent route (Le Roy and Wrana, 2005). In the classic clathrin-dependent endocytic pathway, soluble clathrins assemble into a polygonal lattice at the plasma membrane to form coated pits that bud and pinch off from the membrane in a dynamin-dependent manner and give rise to clathrin-coated vesicles (Kirchhausen, 2000; Bonifacino and Lippincott-Schwartz, 2003). In contrast, nonclathrin-mediated internalization is not inhibited by interfering with clathrin-mediated endocytosis but is sensitive to cholesterol depletion. We have shown that agrin induces rapid translocation of MuSK into lipid rafts, which is required for downstream signaling and AChR clustering (Zhu et al., 2006). Furthermore, agrin signaling and induced AChR clustering were sensitive to nonacute cholesterol depletion by MCD. Raft-dependent endocytosis is sensitive to inhibition of dynamin function. The GTPase activity of dynamin is required for the budding of lipid rafts from purified endothelial plasma membranes (Oh et al., 1998). Microinjection of anti-dynamin antibodies, or expression of a dominant-negative dynamin K44A mutant (K44A) deficient in GTP hydrolysis, prevents the raftmediated internalization of various molecules (Henley et al., 1998; Dessy et al., 2000; Lamaze et al., 2001; Le et al., 2002; Pelkmans and Helenius, 2002). Consistently, we show in the present study that MuSK endocytosis and AChR clustering was attenuated by the dynamin mutant K44A (Fig. 2), providing evidence that MuSK internalization may be raft dependent. It should also be noted that despite significant MuSK endocytosis, the endocytosis of AChRs was barely detectable, even in the presence of agrin stimulation. Therefore, the effects of the dynamin mutant K44A (Fig. 2) and NEM (Fig. 5) on AChR clustering are believed to be specific to MuSK endocytosis. Together, our results indicate a necessary role of induced MuSK endocytosis in agrin signaling.

Although AChR endocytosis is barely detectable in cultured muscle cells, a significant number of AChRs recycle back into the postsynaptic membrane in vivo (Akaaboune et al., 1999; Bruneau 
et al., 2005; Bruneau and Akaaboune, 2006). Our present study suggests the importance of agrin-induced MuSK endocytosis in AChR clustering. It would be interesting to investigate whether NSF is involved in AChR recycling in vivo. Nevertheless, the differential endocytosis of MuSK and AChR in cultured muscle cells could suggest that AChR endocytosis may be mediated by a different mechanism.

\section{References}

Akaaboune M, Culican SM, Turney SG, Lichtman JW (1999) Rapid and reversible effects of activity on acetylcholine receptor density at the neuromuscular junction in vivo. Science 286:503-507.

Beattie EC, Howe CL, Wilde A, Brodsky FM, Mobley WC (2000) NGF signals through TrkA to increase clathrin at the plasma membrane and enhance clathrin-mediated membrane trafficking. J Neurosci 20:7325-7333.

Bonifacino JS, Lippincott-Schwartz J (2003) Coat proteins: shaping membrane transport. Nat Rev Mol Cell Biol 4:409-414.

Borges LS, Ferns M (2001) Agrin-induced phosphorylation of the acetylcholine receptor regulates cytoskeletal anchoring and clustering. J Cell Biol 153:1-12.

Bruneau E, Sutter D, Hume RI, Akaaboune M (2005) Identification of nicotinic acetylcholine receptor recycling and its role in maintaining receptor density at the neuromuscular junction in vivo. J Neurosci 25:9949-9959.

Bruneau EG, Akaaboune M (2006) The dynamics of recycled acetylcholine receptors at the neuromuscular junction in vivo. Development 133:4485-4493.

Campanelli JT, Hoch W, Rupp F, Kreiner T, Scheller RH (1991) Agrin mediates cell contact-induced acetylcholine receptor clustering. Cell 67:909-916.

Carpentier JL (1994) Insulin receptor internalization: molecular mechanisms and physiopathological implications. Diabetologia 37:S117-S124.

Ceresa BP, Schmid SL (2000) Regulation of signal transduction by endocytosis. Curr Opin Cell Biol 12:204-210.

Cheusova T, Khan MA, Schubert SW, Gavin AC, Buchou T, Jacob G, Sticht H, Allende J, Boldyreff B, Brenner HR, Hashemolhosseini S (2006) Casein kinase 2-dependent serine phosphorylation of MuSK regulates acetylcholine receptor aggregation at the neuromuscular junction. Genes Dev 20:1800-1816.

Coppolino MG, Kong C, Mohtashami M, Schreiber AD, Brumell JH, Finlay BB, Grinstein S, Trimble WS (2001) Requirement for $\mathrm{N}$-ethylmaleimide-sensitive factor activity at different stages of bacterial invasion and phagocytosis. J Biol Chem 276:4772-4780

Danino D, Hinshaw JE (2001) Dynamin family of mechanoenzymes. Curr Opin Cell Biol 13:454-460

Dessy C, Kelly RA, Balligand JL, Feron O (2000) Dynamin mediates caveolar sequestration of muscarinic cholinergic receptors and alteration in $\mathrm{NO}$ signaling. EMBO J 19:4272-4280.

Di Fiore PP, De Camilli P (2001) Endocytosis and signaling. an inseparable partnership. Cell 106:1-4.

Ehlers MD (2000) Reinsertion or degradation of AMPA receptors determined by activity-dependent endocytic sorting. Neuron 28:511-525.

Ferns MJ, Campanelli JT, Hoch W, Scheller RH, Hall Z (1993) The ability of
B

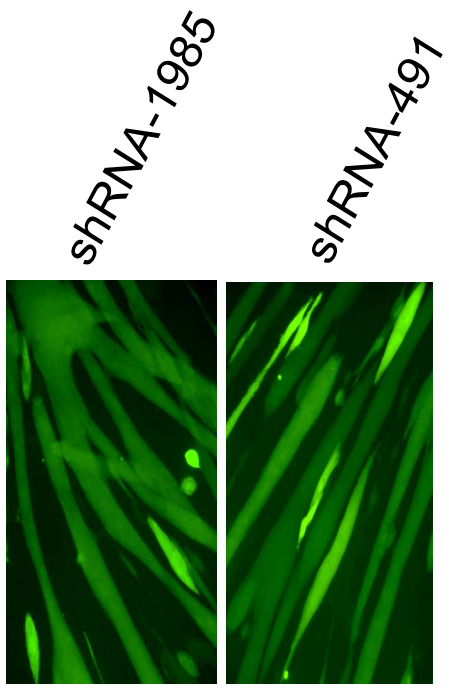

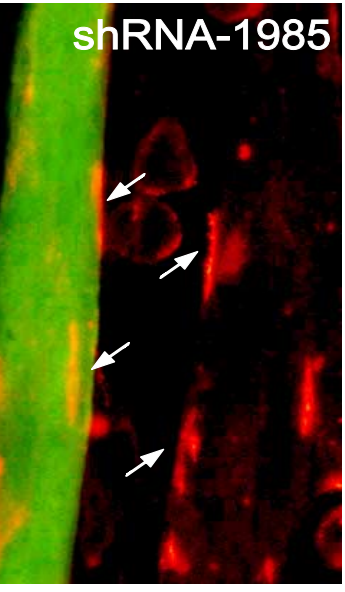

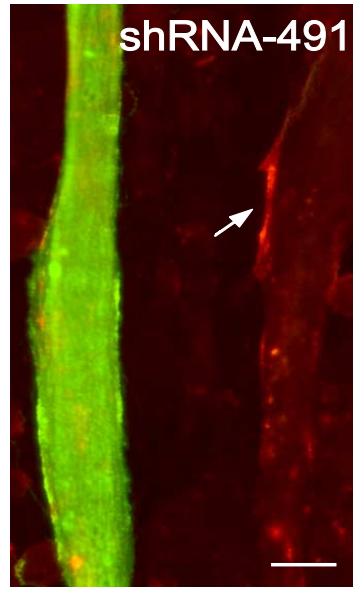

Figure 6. NSF is required for agrin-induced AChR clusters. $A$, NSF shRNAs suppress expression of NSF, but not MuSK. C2C12 cells were transfected with NSF shRNA-491,-824, -1985 , or control shRNA-scramble. Lysates of transfectants were subjected to immunoblotting using indicated antibodies. Mgn, Myogenin. B, NSF shRNAs did not affect myotube formation. C2C12 cells were . A rin-induced AChR clustering was inhibited by NSF shRNAs. 2 212 myoblasts were transfected with alone or together with individual shRNA constructs (pEGFP:shRNA, 1:20). Myotubes were stimulated with agrin and scored for AChR clusters. Images of representative experiments that were repeated three times with similar results are shown. AChR clusters are indicated by arrows. Scale bar, $20 \mu \mathrm{m}$.

agrin to cluster AChRs depends on alternative splicing and on cell surface proteoglycans. Neuron 11:491-502.

Finn AJ, Feng G, Pendergast AM (2003) Postsynaptic requirement for Abl kinases in assembly of the neuromuscular junction. Nat Neurosci 6:717-723.

Gautam M, Noakes PG, Moscoso L, Rupp F, Scheller RH, Merlie JP, Sanes JR (1996) Defective neuromuscular synaptogenesis in agrin-deficient mutant mice. Cell 85:525-535.

Glass DJ, Bowen DC, Stitt TN, Radziejewski C, Bruno J, Ryan TE, Gies DR, Shah S, Mattsson K, Burden SJ, DiStefano PS, Valenzuela DM, DeChiara TM, Yancopoulos GD (1996) Agrin acts via a MuSK receptor complex. Cell 85:513-523.

Henley JR, Krueger EW, Oswald BJ, McNiven MA (1998) Dynaminmediated internalization of caveolae. J Cell Biol 141:85-99. 
A

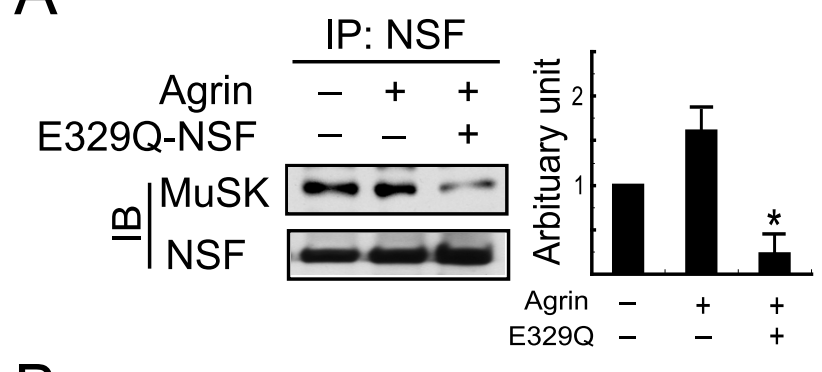

B
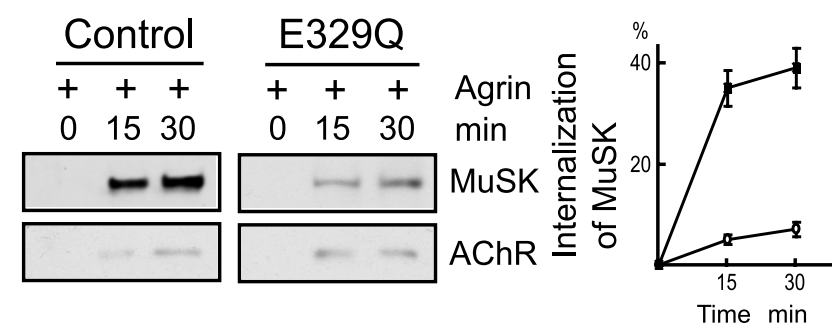

C

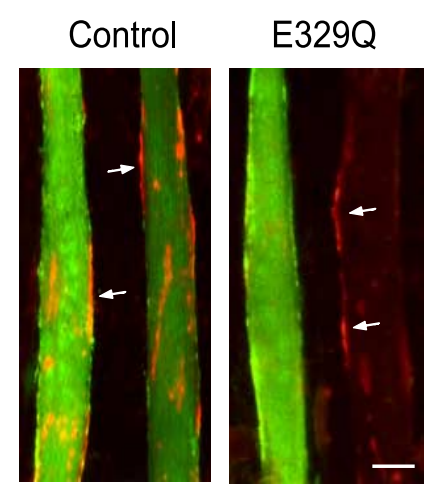

D

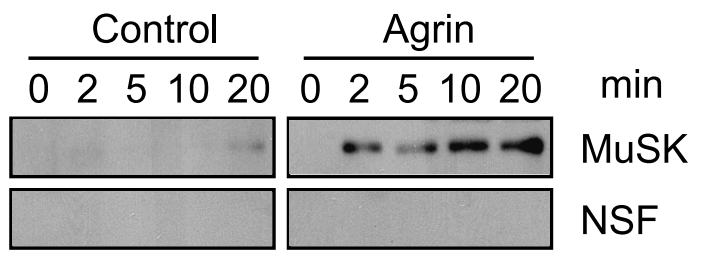

Figure 7. Mutant NSF attenuated AChR clustering. $A$, Mutant NSF inhibited MuSK-NSF interaction. C2C12 cells were transfected with wild-type and E329Q-NSF; fully differentiated myotubes were treated with agrin ( $5 \mathrm{~nm} ; 30 \mathrm{~min}$ ). Lysates were subjected to immunoprecipitation (IP) with anti-NSF antibody, and the membrane was probed with MuSK antibody. B, E329Q-NSF inhibited MuSK endocytosis. C2C12 cells were transfected with wild-type/E3290NSF, and MuSK endocytosis was assayed as in Figure 1. C, Agrin-induced AChR clustering was inhibited by E3290 mutant. C2C12 myoblasts were transfected with pEGFP alone or together with wild-type/E329Q-NSF. Three days later, fully differentiated myotubes were stimulated without or with agrin (1 nм; 16h) and scored for AChR clusters. Scale bar, $20 \mu \mathrm{m}$. D, NSF was not detectable with endocytosed MuSK. MuSK endocytosis was assayed as in Figure 1.

Herbst R, Burden SJ (2000) The juxtamembrane region of MuSK has a critical role in agrin-mediated signaling. EMBO J 19:67-77.

Howe CL, Valletta JS, Rusnak AS, Mobley WC (2001) NGF signaling from clathrin-coated vesicles: evidence that signaling endosomes serve as a platform for the Ras-MAPK pathway. Neuron 32:801-814.

Huang YZ, Won S, Ali DW, Wang Q, Tanowitz M, Du QS, Pelkey KA, Yang DJ, Xiong WC, Salter MW, Mei L (2000) Regulation of neuregulin signaling by PSD-95 interacting with ErbB4 at CNS synapses. Neuron 26:443-455.

Jennings CGB, Dyer SM, Burden SJ (1993) Muscle-specific trk-related re-
A

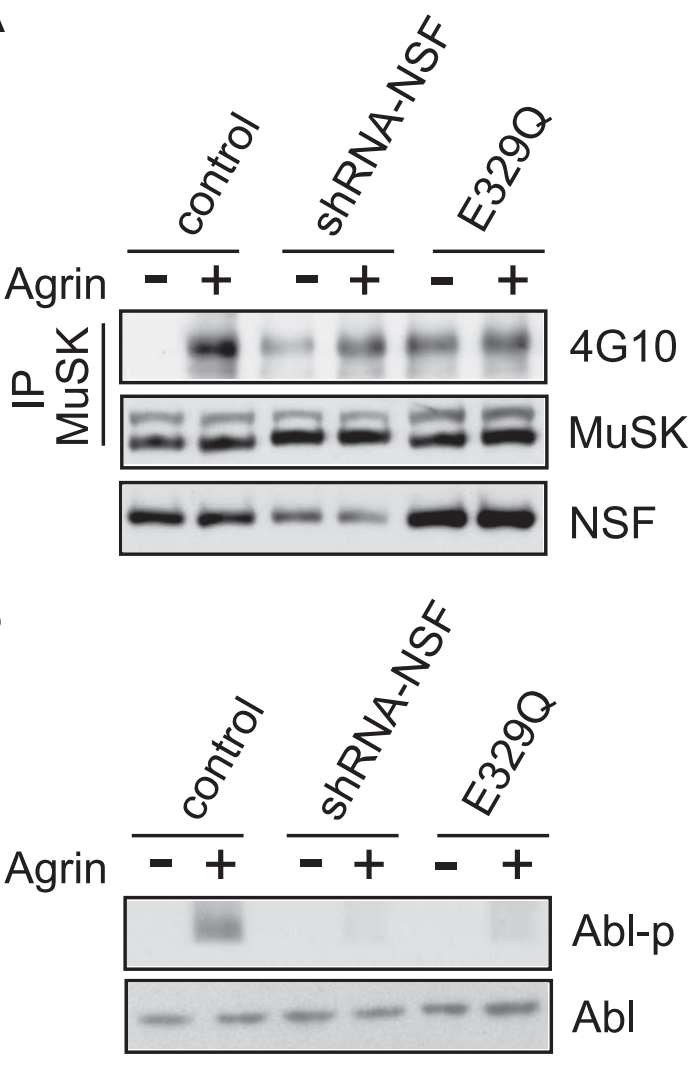

C

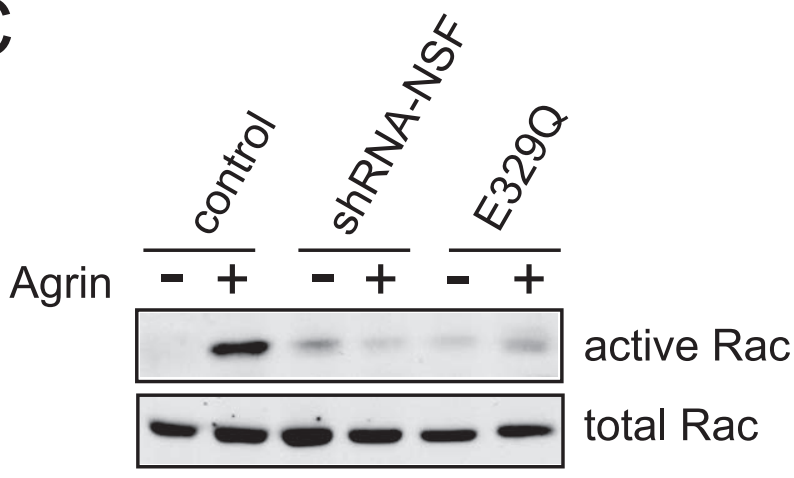

Figure 8. Suppression of NSF expression and mutation of NSF attenuate the agrin/MuSK signaling. $\boldsymbol{A}$, Inhibition of agrin-induced MuSK phosphorylation by NSF shRNA and E3290. C2C12 cells were transfected with NSF shRNA-491 or E329Q-NSF before stimulation with agrin ( $5 \mathrm{~nm} ; 30 \mathrm{~min}$ ). MuSK was immunoprecipitated (IP) and examined for tyrosine phosphorylation by immunoblotting with anti-phosphotyrosine antibody (top panel). The bottom panel shows equal amount of precipitated MuSK. B, Effect on Abl tyrosine phosphorylation. C2C12 cells were transfected with NSF shRNA-491 or E329Q before stimulation with agrin ( $5 \mathrm{~nm} ; 30 \mathrm{~min}$ ). Phosphorylation of Abl was examined with anti-Abl phosphorylation-specific antibody. C, Inhibition of Rac activation. Active Rac was purified by GST-PBD (Cdc42/Rac-binding domain of PAK fused to GST) immobilized on beads and revealed by Western blotting with anti-Rac antibody (top panel). The bottom panel shows equal amounts of Rac.

ceptor with a kringle domain defines a distinct class of receptor tyrosine kinases. Proc Natl Acad Sci USA 90:2895-2899.

Jing S, Tapley P, Barbacid M (1992) Nerve growth factor mediates signal transduction through trk homodimer receptors. Neuron 9:1067-1079.

Jones N, Hardy WR, Friese MB, Jorgensen C, Smith MJ, Woody NM, Burden SJ, Pawson T (2007) Analysis of a Shc family adaptor protein, ShcD/ Shc4, that associates with Muscle-Specific Kinase (MuSK). Mol Cell Biol 27:4759-4773.

Kirchhausen T (2000) Clathrin. Annu Rev Biochem 69:699-727.

Lamaze C, Dujeancourt A, Baba T, Lo CG, Benmerah A, Dautry-Varsat A (2001) Interleukin 2 receptors and detergent-resistant membrane do- 
mains define a clathrin-independent endocytic pathway. Mol Cell 7:661-671.

Le PU, Guay G, Altschuler Y, Nabi IR (2002) Caveolin-1 is a negative regulator of caveolae-mediated endocytosis to the endoplasmic reticulum. J Biol Chem 277:3371-3379.

Le Roy C, Wrana JL (2005) Clathrin- and non-clathrin-mediated endocytic regulation of cell signalling. Nat Rev Mol Cell Biol 6:112-126.

Liu Y, Tao YM, Woo RS, Xiong WC, Mei L (2007) Stimulated ErbB4 internalization is necessary for neuregulin signaling in neurons. Biochem Biophys Res Commun 354:505-510.

Lu Z, Je HS, Young P, Gross J, Lu B, Feng G (2007) Regulation of synaptic growth and maturation by a synapse-associated $\mathrm{E} 3$ ubiquitin ligase at the neuromuscular junction. J Cell Biol 177:1077-1089.

Luo Z, Wang Q, Zhou J, Wang J, Liu M, He X, Wynshaw-Boris A, Xiong W, Lu B, Mei L (2002) Regulation of AChR clustering by dishevelled interacting with MuSK and PAK1. Neuron 35:489-505.

Luo ZG, Je HS, Wang Q, Yang F, Dobbins GC, Yang ZH, Xiong WC, Lu B, Mei L (2003) Implication of geranylgeranyltransferase I in synapse formation Neuron 40:703-717.

Malhotra V, Orci L, Glick BS, Block MR, Rothman JE (1988) Role of an $\mathrm{N}$-ethylmaleimide-sensitive transport component in promoting fusion of transport vesicles with cisternae of the Golgi stack. Cell 54:221-227.

McMahan UJ (1990) The agrin hypothesis. Cold Spring Harb Symp Quant Biol 55:407-418.

Nitkin RM, Smith MA, Magill C, Fallon JR, Yao YM, Wallace BG, McMahan UJ (1987) Identification of agrin, a synaptic organizing protein from Torpedo electric organ. J Cell Biol 105:2471-2478.

Oh P, McIntosh DP, Schnitzer JE (1998) Dynamin at the neck of caveolae mediates their budding to form transport vesicles by GTP-driven fission from the plasma membrane of endothelium. J Cell Biol 141:101-114.

Okada K, Inoue A, Okada M, Murata Y, Kakuta S, Jigami T, Kubo S, Shiraishi H, Eguchi K, Motomura M, Akiyama T, Iwakura Y, Higuchi O, Yamanashi Y (2006) The muscle protein Dok-7 is essential for neuromuscular synaptogenesis. Science 312:1802-1805.

Pelkmans L, Helenius A (2002) Endocytosis via caveolae. Traffic 3:311-320.

Riccio A, Pierchala BA, Ciarallo CL, Ginty DD (1997) An NGF-TrkAmediated retrograde signal to transcription factor CREB in sympathetic neurons. Science 277:1097-1100.

Sadasivam G, Willmann R, Lin S, Erb-Vogtli S, Kong XC, Ruegg MA, Fuhrer C (2005) Src-family kinases stabilize the neuromuscular synapse in vivo via protein interactions, phosphorylation, and cytoskeletal linkage of acetylcholine receptors. J Neurosci 25:10479-10493.
Si J, Luo Z, Mei L (1996) Induction of acetylcholine receptor gene expression by ARIA requires activation of mitogen-activated protein kinase. J Biol Chem 271:19752-19759.

Tagaya M, Wilson DW, Brunner M, Arango N, Rothman JE (1993) Domain structure of an N-ethylmaleimide-sensitive fusion protein involved in vesicular transport. J Biol Chem 268:2662-2666.

Valenzuela DM, Stitt TN, DiStefano PS, Rojas E, Mattsson K, Compton DL, Nunez L, Park JS, Stark JL, Gies DR, Thomas S, Le Beau MM, Fernald AA, Copeland NG, Jenkins NA, Burden SJ, Glass DJ, Yancopoulos GD (1995) Receptor tyrosine kinase specific for the skeletal muscle lineage: expression in embryonic muscle, at the neuromuscular junction, and after injury. Neuron 15:573-584.

Vieira AV, Lamaze C, Schmid SL (1996) Control of EGF receptor signaling by clathrin-mediated endocytosis. Science 274:2086-2089.

Wallace BG (1992) Mechanism of agrin-induced acetylcholine receptor aggregation. J Neurobiol 23:592-604.

Wang Y, Pennock SD, Chen X, Kazlauskas A, Wang Z (2004) Plateletderived growth factor receptor-mediated signal transduction from endosomes. J Biol Chem 279:8038-8046.

Ward DM, Leslie JD, Kaplan J (1997) Homotypic lysosome fusion in macrophages: analysis using an in vitro assay. J Cell Biol 139:665-673.

Weston C, Yee B, Hod E, Prives J (2000) Agrin-induced acetylcholine receptor clustering is mediated by the small guanosine triphosphatases Rac and Cdc42. J Cell Biol 150:205-212.

Whiteheart SW, Rossnagel K, Buhrow SA, Brunner M, Jaenicke R, Rothman JE (1994) N-ethylmaleimide-sensitive fusion protein: a trimeric ATPase whose hydrolysis of ATP is required for membrane fusion. J Cell Biol 126:945-954.

Yang XL, Huang YZ, Xiong WC, Mei L (2005) Neuregulin-induced expression of the acetylcholine receptor requires endocytosis of ErbB receptors. Mol Cell Neurosci 28:335-346.

Zhang Y, Moheban DB, Conway BR, Bhattacharyya A, Segal RA (2000) Cell surface Trk receptors mediate NGF-induced survival while internalized receptors regulate NGF-induced differentiation. J Neurosci 20:5671-5678.

Zhou H, Glass DJ, Yancopoulos GD, Sanes JR (1999) Distinct domains of MuSK mediate its abilities to induce and to associate with postsynaptic specializations. J Cell Biol 146:1133-1146.

Zhu D, Xiong WC, Mei L (2006) Lipid rafts serve as a signaling platform for nicotinic acetylcholine receptor clustering. J Neurosci 26:4841-4851. 